\title{
Simulation of Dengue Disease with Control
}

\author{
Nita H. Shah ${ }^{1}$, Foram A. Thakkar ${ }^{2}$, Bijal M. Yeolekar ${ }^{3}$ \\ ${ }^{I}$ Professor, Department of Mathematics, Gujarat University, Ahmedabad, India \\ ${ }^{2}$ Research Scholar, Department of Mathematics, Gujarat University, Ahmedabad, India \\ ${ }^{3}$ Research Scholar, Department of Mathematics, Gujarat University, Ahmedabad, India
}

*Corresponding Author: Nita H. Shah, Professor, Department of Mathematics, Gujarat University, Ahmedabad, India

\begin{abstract}
These Dengue is a disease which is transmitted to humans by a female mosquito named 'Aedes Aegypti'. In this paper, a mathematical model has been developed to study the spread of dengue disease. Population of human, mosquitoes and their eggs are taken into consideration. To study the dynamics of the disease a compartmental model involving non-linear ordinary differential equations has been formulated for human, mosquitoes and egg population. Stability analysis has been carried out at dengue free and endemic equilibrium points. Control in terms of preventive measures, spraying insecticides, and killing their eggs which is food for guppy fish are to be taken/applied to humans, mosquitoes and eggs respectively. Numerical Simulation has been carried out to show the impact of control on different compartments.
\end{abstract}

Keywords: Mathematical model, System of non-linear ordinary differential equations, Dengue, Stability, Control.

\section{INTRODUCTION}

Dengue is a painful devastating mosquito borne disease caused by the dengue virus name 'Aedes Aegypti'. Aedes Aegypti also known as yellow fever mosquito is a mosquito which can spread dengue, chikungunya, Zika etc. and many other diseases. This mosquito has a white marking on its legs and has marking on upper surface of thorax in the form of lyre. This mosquito which was originated first in Africa is now found in all the tropical and subtropical regions over the world [1].

Of these only female mosquitoes bites which she needs to mature her eggs. Mosquitoes are attracted to the chemical compounds like ammonia, carbon dioxide, lactic acid etc. which are emitted by mammals to find a host. On taking blood meal this mosquito can produce an average of 100 to 200 eggs per batch. During a life time they can produce up to five batches. These number of eggs depends on the size of blood meal. The eggs of this mosquito are smooth, long, oval and about 1 millimeter long. Initially, when laid they appear to be white but in few minutes it turns out to be shiny black. They prefer to lay eggs in tree holes, pots, tanks, water cooler, temporary flood areas and a lot at places where rain water is collected. Development of eggs depends upon time. In warmer climate they take about two days, while in cooler climate they may take upon a week to develop. In dry regions, laid eggs can survive more than years. But once submerged in water, they hatch immediately. These viruses are therefore difficult to control. Infected eggs hatch and becomes an infected mosquito. With regards to control the attack of the diseases, a method to reduce a population of mosquito has been prepared in which a guppy fish is placed in water which eats the mosquito larvae [2].

When a mosquito born with dengue virus bites a person, the virus enters into the skin along with its saliva. It enters into the white blood cells and while moving throughout the body they reproduce inside the cells. This production of virus in the body harms many organs of the body such as liver, bone marrow etc. Normally, these mosquito bites at dawn and dusk time and thus can spread infection at any time of the day. When it bites a person with dengue virus in their blood the mosquito becomes infected [3]. It is not a contagious disease. The symptoms of dengue include fever, headache, joint and muscular pain, nausea, vomiting etc. Similar symptoms as those of common cold, vomiting and diarrhea are found in children, though they prove to be mild initially including high fever but can increase the risk of severity. Particularly, there is no vaccine for this disease [4]. Thus, to protect oneself from the bites of infected mosquitoes make use of mosquito repellents and making use of 
spraying insecticides has to be done to kill them. Preventive measures should be taken care off by individuals.

Asmaidi et al. [5] has done their research entitled "A SIR Mathematical Model of Dengue transmission and its simulation" in which he has incorporated temperature as one of the parameter.

This paper has been modelled by considering eggs, mosquitoes and human population as all the three plays an important role in dengue disease. In this paper, human population has been divided into susceptible humans, infected humans, hospitalized humans, recovered humans. Mosquito population has been divided into susceptible mosquitoes and infected mosquitoes. Egg population has been divided into susceptible eggs and infected eggs. Mathematical model for the transmission of dengue disease has been described in Section 2. Section 3, 4 and 5 includes stability, control and simulation for the compartments. Conclusion is described in Section 6.

\section{Mathematical Model}

Here, we formulate a mathematical model for human-mosquito-egg population. The notations with its description for each parameter is given below Table 1.

Table1. Notations and its Parametric Values

\begin{tabular}{|c|c|c|}
\hline Notations & Description & Parametric Values \\
\hline$N_{H}$ & Sample Size of Human population & 1000 \\
\hline$N_{M}$ & Sample Size of Mosquitoes population & 10000 \\
\hline$N_{E}$ & Sample Size of mosquitoes Eggs population & 100000 \\
\hline$S_{H}(t)$ & Number of Susceptible Humans at some instant of time $t$ & 500 \\
\hline$I_{H}(t)$ & Number of Infected Humans at some instant of time $t$ & 150 \\
\hline$H_{H}(t)$ & Number of Susceptible Humans at some instant of time $t$ & 80 \\
\hline$R_{H}(t)$ & Number of Recovered Humans at some instant of time $t$ & 35 \\
\hline$S_{M}(t)$ & Number of Susceptible Mosquitoes at some instant of time $t$ & 4000 \\
\hline$I_{M}(t)$ & Number of Infected Mosquitoes at some instant of time $t$ & 2500 \\
\hline$S_{E}(t)$ & Number of Susceptible Eggs at some instant of time $t$ & 60000 \\
\hline$I_{E}(t)$ & Number of Infected Eggs at some instant of time $t$ & 40000 \\
\hline $\mathrm{B}$ & New Recruitment Rate & 0.0000024 \\
\hline$\mu_{H}$ & Humans Death Rate & 0.035 \\
\hline$\mu_{M}$ & Mosquitoes Death Rate & 0.05 \\
\hline$\mu_{E}$ & Eggs Death Rate & 0.4 \\
\hline$\alpha$ & Disease Induced Death Rate of Humans & 0.001 \\
\hline$\beta_{H}$ & $\begin{array}{l}\text { Transmission rate of humans from susceptible to infection caused by } \\
\text { infected mosquitoes }\end{array}$ & 0.14 \\
\hline$\delta_{H}$ & Transmission rate of humans from infected to hospitalized & 0.6 \\
\hline$\eta_{H}$ & Transmission rate of humans from hospitalized to recovered & 0.3 \\
\hline$\beta_{M}$ & $\begin{array}{l}\text { Transmission rate of mosquitoes from susceptible to infection caused by } \\
\text { infected humans }\end{array}$ & 0.6 \\
\hline$\rho$ & Infected Eggs Hatching Rate & 0.15 \\
\hline$\theta$ & Proportion of infected eggs laid by an infected mosquito & 0.1 \\
\hline$C_{E}$ & Climatic Factor & 0.07 \\
\hline$\gamma_{M}$ & Oviposition Rate & 0.3 \\
\hline$u_{1}$ & Control rates in terms of preventive measures & {$[0,1]$} \\
\hline$u_{2}$ & Control rates in terms of spraying insecticides & {$[0,1]$} \\
\hline$u_{3}$ & Control rate in terms of guppy fish & {$[0,1]$} \\
\hline
\end{tabular}


The transmission diagram of dengue from different compartments is shown in Figure 1.

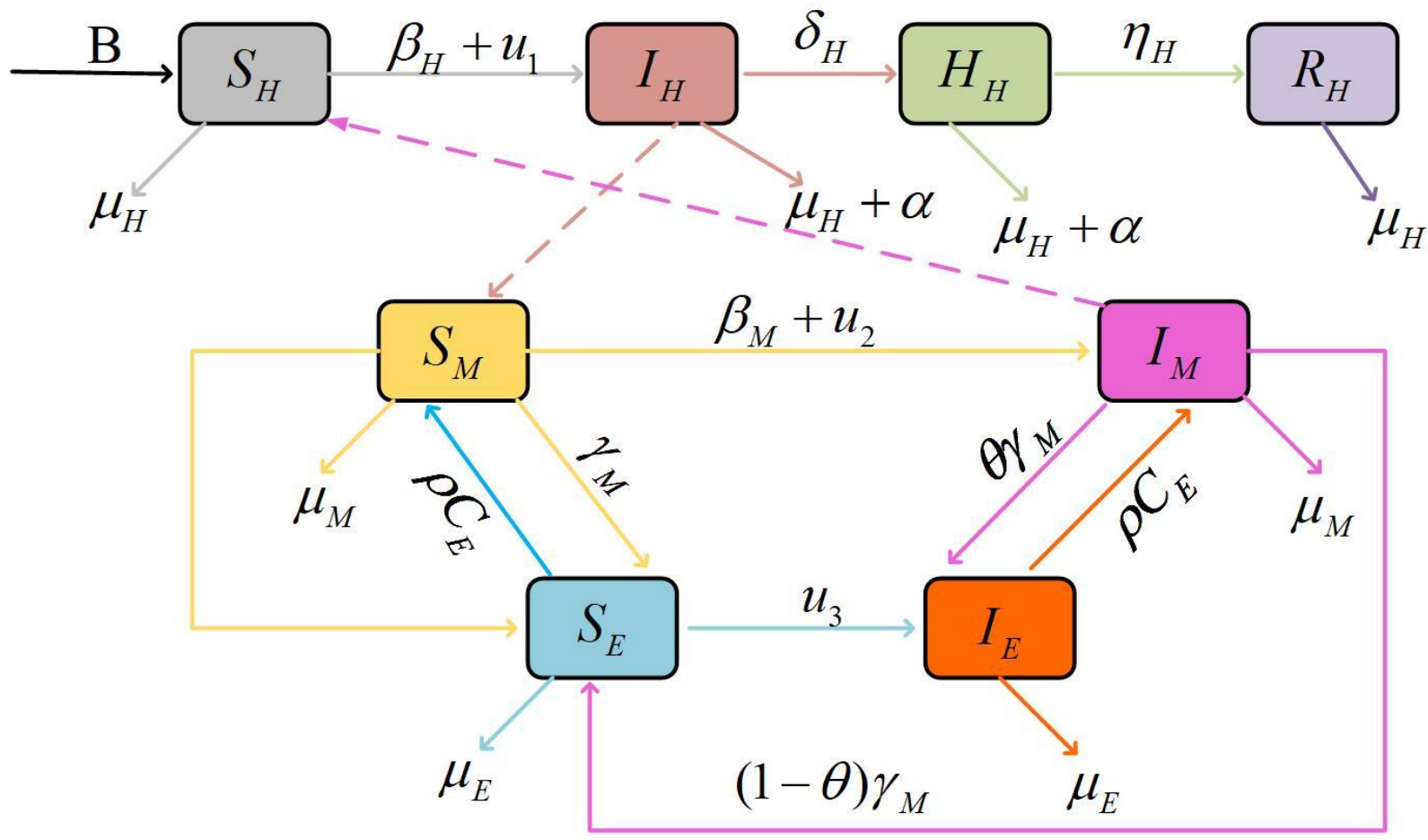

Fig 1.

Infected mosquito $\left(I_{M}\right)$ can infect susceptible $\operatorname{egg}\left(S_{E}\right)$ and susceptible human being $\left(S_{H}\right)$. Also, infected human $\left(I_{H}\right)$ can infect susceptible mosquito $\left(S_{M}\right)$. So, the disease can spread by two ways: infected mosquito and infected human. Susceptible human infects human at the rate $\beta_{H}$ and so total individuals in the infected human compartment are $\beta_{H} \frac{I_{M}}{N_{H}} S_{H}$ and the rate of $\delta_{H}$ infected humans are seeking hospitalization so total individuals in the hospitalized compartment $\left(H_{H}\right)$ are $\delta_{H} I_{H}$. The treatment given in $H_{H}$ compartment makes individual free of dengue disease at the rate $\eta_{H}$ and so total individuals recovered $\left(R_{H}\right)$ from it are $\eta_{H} H_{H}$. Disease induced death occurs in two human compartment namely infected humans and hospitalized humans at the rate $\alpha$. Infected humans coming in contact with a susceptible mosquito makes them infected by the rate $\beta_{M}$. Total infected mosquito population is $\beta_{M} \frac{I_{H}}{N_{H}} S_{M}$. Susceptible eggs and infected eggs $\left(I_{E}\right)$ hatches their infected eggs at the rate $\rho$ depending upon the climatic factor $C_{E}$. Oviposition rate is described by $\gamma_{M}$ and the proportion of infected eggs laid by an infected mosquito is described with the rate $\theta . \mu_{E}$ and $\mu_{M}$ denotes the death of eggs or mosquitoes due to unfavorable conditions may be high temperature or high humidity index, while $\mu_{H}$ denotes natural death rate of humans. Control $u_{1}$ in terms of preventive measures at human population, $u_{2}$ in terms of spraying insecticides for mosquito population, $u_{3}$ to curtail number of infected eggs by use of guppy fish which is an aquatic creature.

So, from the above figure 1 , we have the following set of nonlinear ordinary differential equations describing the causes and cureness of dengue from one compartment to other. 


$$
\begin{aligned}
\frac{d S_{H}}{d t} & =\mathrm{B} N_{H}\left(1-\frac{N_{H}}{K_{H}}\right)-\left(\beta_{H} \frac{I_{M}}{N_{H}}+\mu_{H}\right) S_{H} \\
\frac{d I_{H}}{d t} & =\beta_{H} \frac{I_{M}}{N_{H}} S_{H}-\left(\delta_{H}+\mu_{H}+\alpha\right) I_{H} \\
\frac{d H_{H}}{d t} & =\delta_{H} I_{H}-\left(\eta_{H}+\mu_{H}+\alpha\right) H_{H} \\
\frac{d R_{H}}{d t} & =\eta_{H} H_{H}-\mu_{H} R_{H} \\
\frac{d S_{M}}{d t} & =-\beta_{M} \frac{I_{H}}{N_{H}} S_{M}+\rho C_{E} S_{E}-\left(\mu_{M}+\gamma_{M}\right) S_{M} \\
\frac{d I_{M}}{d t} & =\beta_{M} \frac{I_{H}}{N_{H}} S_{M}+\rho C_{E} I_{E}-\left(\theta \gamma_{M}+(1-\theta) \gamma_{M}+\mu_{M}\right) I_{M} \\
\frac{d S_{E}}{d t} & =\left(\gamma_{M} S_{M}+(1-\theta) \gamma_{M} I_{M}\right)\left(1-\frac{N_{E}}{K_{E}}\right)-\left(\mu_{E}+\rho C_{E}\right) S_{E} \\
\frac{d I_{E}}{d t} & =\theta \gamma_{M} I_{M}\left(1-\frac{N_{E}}{K_{E}}\right)-\left(\mu_{E}+\rho C_{E}\right) I_{E}
\end{aligned}
$$

with $S_{H}+I_{H}+H_{H}+R_{H} \leq N_{H}, S_{M}+I_{M} \leq N_{M}, S_{E}+I_{E} \leq N_{E}$

and $S_{H}, S_{M}, S_{E}>0 ; I_{H}, H_{H}, R_{H}, I_{M}, I_{E} \geq 0$.

Simplification of equation (1) has been carried out by comparing each population to a total population i.e.

$$
S_{h}=\frac{S_{H}}{N_{H}}, I_{h}=\frac{I_{H}}{N_{H}}, H_{h}=\frac{H_{H}}{N_{H}}, R_{h}=\frac{R_{H}}{N_{H}}, S_{m}=\frac{S_{M}}{N_{M}}, I_{m}=\frac{I_{M}}{N_{M}}, S_{e}=\frac{S_{E}}{N_{E}}, I_{e}=\frac{I_{E}}{N_{E}}
$$

To obtain a new set of equations mainly of five dimensions' equation (2) is substituted in equation (1) namely as

$$
\begin{aligned}
& \frac{d S_{h}}{d t}=\mathrm{B}\left(1-\frac{N_{H}}{K_{H}}\right)-\left(z_{1} I_{m}+\mu_{H}\right) S_{h} \\
& \frac{d I_{h}}{d t}=z_{1} I_{m} S_{h}-\left(\delta_{H}+\mu_{H}+\alpha\right) I_{h}
\end{aligned}
$$

Error! Bookmark not defined. $\frac{d H_{h}}{d t}=\delta_{H} I_{h}-\left(\eta_{H}+\mu_{H}+\alpha\right) H_{h}$

$$
\begin{aligned}
& \frac{d I_{m}}{d t}=\beta_{M} I_{h}\left(1-I_{m}\right)+\rho C_{E} z I_{E}-\left(\mu_{M}+\gamma_{M}\right) I_{m} \\
& \frac{d I_{E}}{d t}=\frac{\theta \gamma_{M} I_{m}}{z}\left(1-\frac{N_{E}}{K_{E}}\right)-\left(\mu_{E}+\rho C_{E}\right) I_{E}
\end{aligned}
$$

with $S_{h}+I_{h}+H_{h}+R_{h} \leq 1, S_{m}+I_{m} \leq 1, S_{e}+I_{e} \leq 1$

where $z=\frac{N_{E}}{N_{M}}$ and $z_{1}=N_{M} \cdot \beta_{H}$

The feasible region for the above set of equations (3) is 
$\Lambda=\left\{\left(S_{h}+I_{h}+H_{h}+I_{m}+I_{e}\right) / S_{h}+I_{h}+H_{h}+I_{m}+I_{e} \leq \frac{\mathrm{B}\left(1-\frac{N_{H}}{K_{H}}\right)}{\mu_{H}} ; S_{h}>0, I_{h}, H_{h}, I_{m}, I_{e} \geq 0\right\}$

Thus, Dengue free equilibrium point $\left(E_{0}\right)=\left(S_{h}, I_{h}, H_{h}, I_{m}, I_{e}\right)=\left(\frac{\mathrm{B}\left(1-\frac{N_{H}}{K_{H}}\right)}{\mu_{H}}, 0,0,0,0\right)$.

Now, our actual interest lies in calculating the basic reproduction number which is calculated using the next generation matrix method which is defined as $F V^{-1}$ [6] where $F$ and $V$ both are Jacobian matrices of $\Im$ and $v$ evaluated with respect to infected humans, mosquitoes, eggs and hospitalized humans at the point $E_{0}$.

Let $X=\left(I_{h}, H_{h}, I_{m}, I_{e}, S_{h}\right) . \therefore \frac{d X}{d t}=\mathfrak{I}(X)-v(X)$

where $\mathfrak{I}(X)$ denotes the rate of newly recruited and $v(X)$ denotes the rate of derived recruited which is given as

$$
\Im(X)=\left[\begin{array}{c}
z_{1} I_{m} S_{h} \\
0 \\
0 \\
0 \\
0
\end{array}\right] \text { and } v(X)=\left[\begin{array}{c}
\left(\delta_{H}+\mu_{H}+\alpha\right) I_{h} \\
-\delta_{H} I_{h}+\left(\eta_{H}+\mu_{H}+\alpha\right) H_{h} \\
-\beta_{M} I_{h}\left(1-I_{m}\right)-\rho C_{E} z I_{e}+\left(\mu_{M}+\gamma_{M}\right) I_{m} \\
-\frac{\theta \gamma_{M} I_{m}}{z}\left(1-\frac{N_{E}}{K_{E}}\right)+\left(\mu_{E}+\rho C_{E}\right) I_{e} \\
-\mathrm{B}\left(1-\frac{N_{H}}{K_{H}}\right)+\left(z_{1} I_{m}+\mu_{H}\right) S_{h}
\end{array}\right]
$$

Now, the derivative of $\mathfrak{I}$ and $v$ evaluated at a dengue free equilibrium point $\left(E_{0}\right)$ gives matrices $F$ and $V$ of order $5 \times 5$ which is defined as

$$
\begin{aligned}
& F=\left[\frac{\partial \mathfrak{I}_{i}\left(E_{0}\right)}{\partial X_{j}}\right] V=\left[\frac{\partial v_{i}\left(E_{0}\right)}{\partial X_{j}}\right] \text { for } i, j=1,2,3,4,5 \\
& \text { So, } F=\left[\begin{array}{ccccc}
0 & 0 & \frac{z_{1} \mathrm{~B}\left(N_{H}-K_{H}\right)}{\mu_{H} K_{H}} & 0 & 0 \\
0 & 0 & 0 & 0 & 0 \\
0 & 0 & 0 & 0 & 0 \\
0 & 0 & 0 & 0 & 0 \\
0 & 0 & 0 & 0 & 0
\end{array}\right] \text { and } \\
& V=\left[\begin{array}{ccccc}
\delta_{H}+\mu_{H}+\alpha & 0 & 0 & 0 \\
-\delta_{H} & \eta_{H}+\mu_{H}+\alpha & 0 & 0 \\
-\beta_{M} & 0 & \mu_{M}+\gamma_{M} & -\rho C_{E} z & 0 \\
0 & 0 & -\frac{\theta \gamma_{M}}{z}\left(1-\frac{N_{E}}{K_{E}}\right) & \mu_{E}+\rho C_{E} & 0 \\
0 & 0 & \frac{z_{1} \mathrm{~B}\left(N_{H}-K_{H}\right)}{\mu_{H} K_{H}} & 0 & \mu_{H}
\end{array}\right]
\end{aligned}
$$


where $V$ is non-singular matrix. Thus, the basic reproduction number $R_{0}$ which is the spectral radius of matrix $F V^{-1}$ is given as

$$
R_{0}=\frac{z_{1} \mathrm{~B}\left(K_{H}-N_{H}\right)\left(\mu_{E}+\rho C_{E}\right) \beta_{M} K_{E}}{\mu_{H} K_{H}\left(\delta_{H}+\mu_{H}+\alpha\right)\left[\rho C_{E} \gamma_{M}\left(N_{E} \theta+(1-\theta) K_{E}\right)+\mu_{M} C_{E} K_{E} \rho+\mu_{E} K_{E}\left(\mu_{M}+\gamma_{M}\right)\right]}
$$

On equating set of equations (3) to zero, an endemic equilibrium point $\left(E^{*}\right)$ is obtained which is as follows:

$E^{*}=\left(S_{h}^{*}, I_{h}{ }^{*}, H_{h}{ }^{*}, I_{m}{ }^{*}, I_{e}^{*}\right)$ where

$$
\begin{aligned}
& S_{h}^{*}=\frac{\mathrm{B}\left(K_{H}-N_{H}\right)}{K_{H}\left(z_{1} I_{m}{ }^{*}+\mu_{H}\right)}, I_{h}{ }^{*}=\frac{z_{1} I_{m}{ }^{*} S_{h}{ }^{*}}{\mu_{H}+\alpha+\delta_{H}}, \\
& H_{h}{ }^{*}=\frac{I_{m}{ }^{*} \delta_{H} z_{1} S_{h}{ }^{*}}{K_{H}\left[\mu_{H}\left(\mu_{H}+2 \alpha+\delta_{H}+\eta_{H}\right)+\alpha\left(\alpha+\delta_{H}+\eta_{H}\right) \delta_{H} \eta_{H}\right]}, \\
& I_{m}{ }^{*}=\frac{\rho C_{E} I_{e}^{*} z+\beta_{M} I_{h}{ }^{*}}{\beta_{M} I_{h}{ }^{*}+\mu_{M}+\gamma_{M}}, I_{e}^{*}=\frac{\theta \gamma_{M} I_{m}{ }^{*}\left(K_{E}-N_{E}\right)}{z K_{E}\left(\rho C_{E}+\mu_{E}\right)}
\end{aligned}
$$

\section{Stability ANAlysis}

In this section, the local and global stability at $E_{0}$ and $E^{*}$ using the linearization method and matrix analysis are to be studied.

\subsection{Local Stability}

Theorem 3.1.1 (stability at $E_{0}$ ) If $R_{0}<1$ then a system is locally asymptotically stable at dengue free equilibrium point $E_{0}$. If $R_{0}>1$ then it is unstable.

Proof: Jacobian Matrix of the system evaluated at point $E_{0}$ is

$$
J\left(E_{0}\right)=\left[\begin{array}{ccccc}
-\mu_{H} & 0 & 0 & -\frac{z_{1} \mathrm{~B}\left(N_{H}-K_{H}\right)}{\mu_{H} K_{H}} & 0 \\
0 & -\left(\delta_{H}+\mu_{H}+\alpha\right) & 0 & \frac{z_{1} \mathrm{~B}\left(N_{H}-K_{H}\right)}{\mu_{H} K_{H}} & 0 \\
0 & \delta_{H} & -\left(\eta_{H}+\mu_{H}+\alpha\right) & 0 & 0 \\
0 & \beta_{M} & 0 & -\left(\mu_{M}+\gamma_{M}\right) & \rho C_{E} z \\
0 & 0 & 0 & \frac{\theta \gamma_{M}}{z}\left(1-\frac{N_{E}}{K_{E}}\right) & -\left(\mu_{E}+\rho C_{E}\right)
\end{array}\right]
$$

The eigenvalues of the characteristic equation are $\lambda_{1}=-\mu_{H}<0, \lambda_{2}=-\left(\eta_{H}+\mu_{H}+\alpha\right)$ and $\lambda_{3}, \lambda_{4}, \lambda_{5}$ satisfies the equation $a_{0} \lambda^{3}+a_{1} \lambda^{2}+a_{2} \lambda+a_{3}=0$ where

$$
\begin{aligned}
& a_{0}=1>0 \\
& a_{1}=\rho C_{E}+\mu_{E}+\mu_{H}+\alpha+\delta_{H}+\gamma_{M}>0
\end{aligned}
$$


$a_{2}=\left\{\begin{array}{l}\rho C_{E}\left(\mu_{H}+\mu_{M}+\delta_{H}+\alpha+\gamma_{M}(1-\rho)\right) \\ +\mu_{E}\left(\mu_{H}+\mu_{M}+\alpha+\delta_{H}+\gamma_{M}\right) \\ +\mu_{H}\left(\mu_{M}+\gamma_{M}\right)+\left(\mu_{M}+\gamma_{M}\right)\left(\alpha+\delta_{H}\right)\end{array}\right\}+\left[\frac{\mu_{H} C_{E} K_{H} N_{E} \gamma_{M} \theta \rho-\beta_{M} \mathrm{~B} K_{E} z_{1}\left(K_{H}-N_{H}\right)}{\mu_{H} K_{E} K_{H}}\right]>0$ which is obvious.

$a_{3}=\frac{\left(\delta_{H}+\mu_{H}+\alpha\right)\left[-C_{E} K_{E} \gamma_{M} \theta \rho+C_{E} N_{E} \gamma_{M} \theta \rho+C_{E} K_{E} \mu_{M} \rho+C_{E} K_{E} \gamma_{M} \rho+\mu_{E} \mu_{M} K_{E}+\mu_{E} \gamma_{M} K_{E}\right]\left[1-R_{0}\right]}{K_{E}}$

So, if $R_{0}<1$ then $a_{3}>0$.

Hence, $a_{1}>0, a_{2}>0, a_{3}>0$ and $a_{1} a_{2}-a_{3}>0$ if $R_{0}<1$.

Thus by Routh Hurwitz criteria [7] all the conditions for the stability of $n=3$ are satisfied.

Therefore, the system is locally asymptotically stable at $E_{0}$.

Theorem 3.1.2 (stability at $E^{*}$ ) If $Q U-R T>0$ and $\mu_{E}-X>0$ then a system is locally asymptotically stable at an endemic equilibrium point $E^{*}$.

Proof: Jacobian Matrix of the system evaluated at point $E^{*}$ is

$$
J\left(E^{*}\right)=\left[\begin{array}{ccccc}
-\left(P+\mu_{H}\right) & 0 & 0 & -T & 0 \\
P & -Q & 0 & T & 0 \\
0 & \delta_{H} & -S & 0 & 0 \\
0 & R & 0 & -U & X z \\
0 & 0 & 0 & V & -\left(X+\mu_{E}\right)
\end{array}\right]
$$

where

$$
\begin{aligned}
& P=z_{1} I_{m}^{*}, Q=\delta_{H}+\mu_{H}+\alpha, R=\beta_{M}\left(1-I_{m}^{*}\right), S=\eta_{H}+\mu_{H}+\alpha, \\
& T=z_{1} S_{h}^{*}, U=\beta_{M} I_{h}^{*}+\mu_{M}+\gamma_{M}, V=\frac{\theta \gamma_{M}}{z}\left(1-\frac{N_{E}}{K_{E}}\right), X=\rho C_{E}
\end{aligned}
$$

The eigenvalues of the characteristic equation are $\lambda_{1}=-S<0$ and $\lambda_{2}, \lambda_{3}, \lambda_{4}, \lambda_{5}$ satisfies the equation $a_{0} \lambda^{4}+a_{1} \lambda^{3}+a_{2} \lambda^{2}+a_{3} \lambda+a_{4}=0$ where

$a_{0}=1>0$

$a_{1}=\mu_{E}+X+U+P+Q+\mu_{H}>0$

$a_{2}=\mu_{E} \mu_{H}+\mu_{E} P+\mu_{E} Q+\mu_{E} U+\mu_{H} Q+\mu_{H} U+\mu_{H} X+P Q+P U+P X+Q U+Q X+U X-V X z-R T>0$ which is also obvious.

$$
\begin{aligned}
a_{3}= & \mu_{E} \mu_{H} Q+\mu_{E} \mu_{H} U+\mu_{E} P Q+\mu_{H} Q X+\mu_{H} U X-V X z\left(\mu_{H}+P+Q\right) \\
& +P U\left(\mu_{E}-X\right)+(Q U-R T)\left\{\mu_{E}+\mu_{H}+X\right\}+P Q U+P Q X
\end{aligned}
$$

So, if $Q U-R T>0$ and $\mu_{E}-X>0$ then $a_{3}>0$ and $-V X z\left(\mu_{H}+P+Q\right)$ is very small term as compared to other terms and consist of a very negligible value.

$$
a_{4}=\mu_{E} P Q U+P Q U X+(Q U-R T)\left\{\mu_{E} \mu_{H}+\mu_{H} X\right\}-V Q X z\left(\mu_{H}+P\right)
$$


But as $\operatorname{VQXz}\left(\mu_{H}+P\right)$ is very small term. So, if $Q U-R T>0$ then $a_{4}>0$.

Thus, $a_{1}>0, a_{2}>0, a_{3}>0, a_{4}>0$ and $a_{1} a_{2} a_{3}>a_{3}^{2}+a_{1}^{2} a_{4}$ if $Q U-R T>0$.

Hence, all the conditions of Routh Hurwitz criteria for the stability are satisfied for $n=4$ so the system is locally asymptotically stable at $E^{*}$.

\subsection{Global Stability}

Theorem 3.2.1 (stability at $E_{0}$ ) If $\frac{z_{1} \mathrm{~B}\left(N_{H}-K_{H}\right)}{\mu K_{H}}<\frac{\theta \gamma_{M}}{z}\left(1-\frac{N_{E}}{K_{E}}\right)$ then a system is globally asymptotically stable at dengue free equilibrium point $E_{0}$.

Proof: Consider a Lyapunov function

$$
\begin{aligned}
L(t)= & I_{h}(t)+H_{h}(t)+I_{m}(t)+I_{e}(t) \\
\therefore L^{\prime}(t)= & -\left(\mu_{H}+\alpha\right)\left(I_{h}+H_{h}\right)-\left(\mu_{E}+\rho C_{E}-\theta C_{E} z\right) I_{e}-\left(\mu_{M}+\gamma_{M}+\beta_{M} I_{h}\right) I_{m} \\
& +\beta_{M} I_{h}+I_{m}\left(z_{1} S_{h}+\frac{\theta \gamma_{M}}{z}\left(1-\frac{N_{E}}{K_{E}}\right)\right)
\end{aligned}
$$

So, if $z_{1} S_{h}<\frac{\theta \gamma_{M}}{z}\left(1-\frac{N_{E}}{K_{E}}\right)$ that is $\frac{z_{1} \mathrm{~B}\left(N_{H}-K_{H}\right)}{\mu K_{H}}<\frac{\theta \gamma_{M}}{z}\left(1-\frac{N_{E}}{K_{E}}\right)$ as $E_{0} \in \Lambda$ and $\mu$ is non negative then $L^{\prime}(t)<0$.

And if $I_{h}=H_{h}=I_{m}=I_{e}=0$ then $L^{\prime}(t)=0$.

Hence, $L^{\prime}(t)=0$ is $E_{0}$.

So, by LaSalle's Invariance Principle [8], every solution of system (1), with initial conditions in $\Lambda$, approaches $E_{0}$ as $t \rightarrow \infty$.

Thus, a system is globally asymptotically stable at point $E_{0}$.

Theorem 3.2.2 (stability at $E^{*}$ ) The system is globally asymptotically stable at endemic equilibrium point $E^{*}$.

Proof: Consider a Lyapunov function

$$
\begin{aligned}
L(t)= & \frac{1}{2}\left[\left\{S_{h}(t)-S_{h}{ }^{*}(t)\right\}+\left\{I_{h}(t)-I_{h}{ }^{*}(t)\right\}+\left\{H_{h}(t)-H_{h}{ }^{*}(t)\right\}+\left\{I_{m}(t)-I_{m}{ }^{*}(t)\right\}+\left\{I_{e}(t)-I_{e}{ }^{*}(t)\right\}\right]^{2} \\
\therefore L^{\prime}(t)= & {\left[\left\{S_{h}(t)-S_{h}{ }^{*}(t)\right\}+\left\{I_{h}(t)-I_{h}{ }^{*}(t)\right\}+\left\{H_{h}(t)-H_{h}{ }^{*}(t)\right\}+\left\{I_{m}(t)-I_{m}{ }^{*}(t)\right\}+\left\{I_{e}(t)-I_{e}{ }^{*}(t)\right\}\right] } \\
& {\left[S_{h}{ }^{\prime}+I_{h}{ }^{\prime}+H_{h}{ }^{\prime}+I_{m}{ }^{\prime}+I_{e}{ }^{\prime}\right] }
\end{aligned}
$$

On using $\mathrm{B}=\mu_{H} S_{H}{ }^{*}+\mu_{H} I_{H}{ }^{*}+\mu_{H} H_{H}{ }^{*}+\mu_{M} I_{m}{ }^{*}+\mu_{E} I_{e}{ }^{*}$ we have

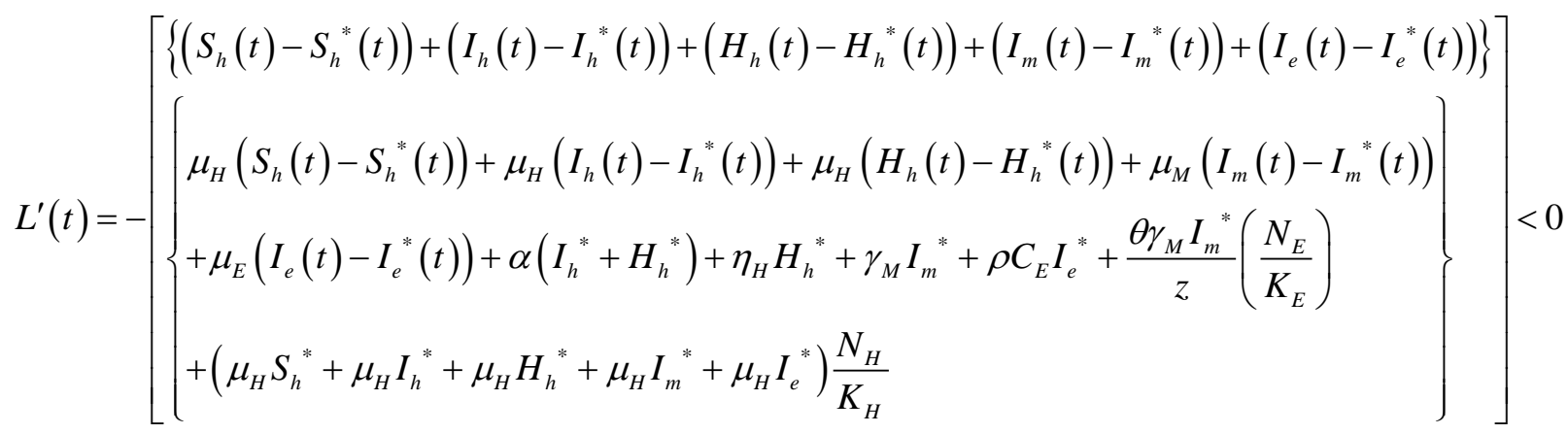

Hence, the system is globally asymptotically stable at $E^{*}$. 


\section{Optimal Control Model}

In this section a control function has been implemented to decrease the spread of dengue disease in human population. The objective function along with the optimal control variable is given by

$$
J\left(u_{i}, \Omega\right)=\int_{0}^{T}\left(\begin{array}{l}
A_{1} S_{H}{ }^{2}+A_{2} I_{H}{ }^{2}+A_{3} H_{H}{ }^{2}+A_{4} R_{H}{ }^{2}+A_{5} S_{M}{ }^{2}+A_{6} I_{M}{ }^{2} \\
+A_{7} S_{E}{ }^{2}+A_{8} I_{E}{ }^{2}+w_{1} u_{1}{ }^{2}+w_{2} u_{2}{ }^{2}+w_{3} u_{3}{ }^{2}
\end{array}\right) d t
$$

where $\Omega$ denotes the set of all compartmental variables, $A_{1}, A_{2}, A_{3}, A_{4}, A_{5}, A_{6}, A_{7}, A_{8}$ denotes nonnegative weight constants for the compartments $S_{H}, I_{H}, H_{H}, R_{H}, S_{M}, I_{M}, S_{E}, I_{E}$ respectively and $w_{1}, w_{2}, w_{3}$ are the weight constant for the control variable $u_{1}, u_{2}, u_{3}$ respectively.

The weights $w_{1}, w_{2}$ and $w_{3}$ which are constant parameters for $u_{1}, u_{2}$ and $u_{3}$ will standardized the optimal control condition.

Now, we will calculate the value of control variables $u_{1}, u_{2}$ and $u_{3}$ from $t=0$ to $t=T$ such that

$$
J\left(u_{1}(t), u_{2}(t), u_{3}(t)\right)=\min \left\{J\left(u_{i}^{*}, \Omega\right) / u_{1}, u_{2}, u_{3} \in \phi\right\}
$$

where $\phi=$ smooth function on the interval $[0,1]$.

Using, Fleming and Rishel results [9], the optimal control denoted by $u_{i}^{*}$ is obtained by collecting all the integrands of the objective function (2) using the lower bounds and upper bounds of the both the control variables respectively.

Using Pontrygin's principle [10], we construct a Lagrangian function consisting of state equation and adjoint variables $A_{V}=\left(\lambda_{S_{H}}, \lambda_{I_{H}}, \lambda_{H_{H}}, \lambda_{R_{H}}, \lambda_{S_{M}}, \lambda_{I_{M}}, \lambda_{S_{E}}, \lambda_{I_{E}}\right)$ which is as follows:

$$
\begin{aligned}
L\left(\Omega, A_{V}\right)= & A_{1} S_{H}{ }^{2}+A_{2} I_{H}^{2}+A_{3} H_{H}^{2}+A_{4} R_{H}^{2}+A_{5} S_{M}^{2}+A_{6} I_{M}^{2}+A_{7} S_{E}^{2}+A_{8} I_{E}^{2}+w_{1} u_{1}^{2}+w_{2} u_{2}^{2}+w_{3} u_{3}^{2} \\
& +\lambda_{S_{H}}\left[\mathrm{~B} N_{H}\left(1-\frac{N_{H}}{K_{H}}\right)-\left(\beta_{H} \frac{I_{M}}{N_{H}}+\mu_{H}\right) S_{H}-u_{1} S_{H}\right] \\
& +\lambda_{I_{H}}\left[\beta_{H} \frac{I_{M}}{N_{H}} S_{H}-\left(\delta_{H}+\mu_{H}+\alpha\right) I_{H}+u_{1} S_{H}\right] \\
& +\lambda_{H_{H}}\left[\delta_{H} I_{H}-\left(\eta_{H}+\mu_{H}+\alpha\right) H_{H}\right] \\
& +\lambda_{R_{H}}\left[\eta_{H} H_{H}-\mu_{H} R_{H}\right] \\
& +\lambda_{S_{M}}\left[-\beta_{M} \frac{I_{H}}{N_{H}} S_{M}+\rho C_{E} S_{E}-\left(\mu_{M}+\gamma_{M}+u_{2}\right) S_{M}\right] \\
& +\lambda_{I_{M}}\left[\beta_{M} \frac{I_{H}}{N_{H}} S_{M}+\rho C_{E} I_{E}-\left(\theta \gamma_{M}+(1-\theta) \gamma_{M}+\mu_{M}\right) I_{M}+u_{2} S_{M}\right] \\
& +\lambda_{S_{E}}\left[\left(\gamma_{M} S_{M}+(1-\theta) \gamma_{M} I_{M}\right)\left(1-\frac{N_{E}}{K_{E}}\right)-\left(\mu_{E}+\rho C_{E}\right) S_{E}-u_{3} S_{E}\right] \\
& +\lambda_{I_{E}}\left[\theta \gamma_{M} I_{M}\left(1-\frac{N_{E}}{K_{E}}\right)-\left(\mu_{E}+\rho C_{E}\right) I_{E}+u_{3} S_{E}\right]
\end{aligned}
$$

Now, the partial derivative of the Lagrangian function with respect to each variable of the compartment gives us the adjoint equation such that 


$$
\begin{aligned}
\dot{\lambda}_{S_{H}} & =-\frac{\partial L}{\partial S_{H}} \\
& =-2 A_{1} S_{H}+\left(\beta_{H} \frac{I_{M}}{N_{H}}+u_{1}\right)\left(\lambda_{S_{H}}-\lambda_{I_{H}}\right)+\mu_{H} \lambda_{S_{H}} \\
\dot{\lambda}_{I_{H}} & =-\frac{\partial L}{\partial I_{H}} \\
& =-2 A_{2} I_{H}+\left(\beta_{M} \frac{S_{M}}{N_{H}}\right)\left(\lambda_{S_{M}}-\lambda_{I_{M}}\right)+\delta_{H}\left(\lambda_{I_{H}}-\lambda_{H_{H}}\right)+\left(\mu_{H}+\alpha\right) \lambda_{I_{H}} \\
\dot{\lambda}_{H_{H}} & =-\frac{\partial L}{\partial H_{H}} \\
& =-2 A_{3} H_{H}+\eta_{H}\left(\lambda_{H_{H}}-\lambda_{R_{H}}\right)+\left(\mu_{H}+\alpha\right) \lambda_{H_{H}} \\
\dot{\lambda}_{R_{H}} & =-\frac{\partial L}{\partial R_{H}} \\
& =-2 A_{4} R_{H}+\mu_{H} \lambda_{R_{H}} \\
\dot{\lambda}_{S_{M}} & =-\frac{\partial L}{\partial S_{M}} \\
& =-2 A_{5} S_{M}+\left(\beta_{M} \frac{I_{H}}{N_{H}}+u_{2}\right)\left(\lambda_{S_{M}}-\lambda_{I_{M}}\right)+\gamma_{M}\left(\lambda_{S_{M}}-\lambda_{S_{E}}\right)+\mu_{M} \lambda_{S_{M}} \\
\dot{\lambda}_{I_{E}} & =-\frac{\partial L}{\partial I_{E}} \\
& =-2 A_{8} I_{E}+\rho C_{E}\left(\lambda_{I_{E}}-\lambda_{I_{M}}\right)+\mu_{E} \lambda_{I_{E}} \\
\dot{\lambda}_{I_{M}} & =-\frac{\partial L}{\partial I_{M}} \\
& =-2 A_{6} I_{M}+\left(\beta_{H} \frac{\partial L}{N_{H}}\right)\left(\lambda_{S_{H}}-\lambda_{I_{H}}\right)+\theta \gamma_{M}\left(1-\frac{N_{E}}{K_{E}}\right)\left(\lambda_{S_{E}}-\lambda_{I_{E}}\right)+\left(\mu_{M}+\gamma_{M}\right) \lambda_{I_{M}}-\gamma_{M} \lambda_{S_{E}}\left(1-\frac{N_{E}}{K_{E}}\right) \\
& \left.=-\lambda_{S_{E}}-\lambda_{S_{M}}\right)+u_{3}\left(\lambda_{S_{E}}-\lambda_{I_{E}}\right)+\mu_{E} \lambda_{S_{E}} \\
& \\
& \\
&
\end{aligned}
$$

The necessary conditions for Lagrangian function $L$ to be optimal for control are

$$
\begin{aligned}
& \frac{\partial L}{\partial u_{1}}=2 w_{1} u_{1}-S_{H}\left(\lambda_{S_{H}}-\lambda_{I_{H}}\right)=0 \\
& \frac{\partial L}{\partial u_{2}}=2 w_{2} u_{2}-S_{M}\left(\lambda_{S_{M}}-\lambda_{I_{M}}\right)=0 \\
& \frac{\partial L}{\partial u_{3}}=2 w_{3} u_{3}-S_{E}\left(\lambda_{S_{E}}-\lambda_{I_{E}}\right)=0
\end{aligned}
$$

On solving equation (5), (6) and (7) we get,

$$
u_{1}=\frac{S_{H}\left(\lambda_{S_{H}}-\lambda_{I_{H}}\right)}{2 w_{1}}, u_{2}=\frac{S_{M}\left(\lambda_{S_{M}}-\lambda_{I_{M}}\right)}{2 w_{2}} \text { and } u_{3}=\frac{S_{E}\left(\lambda_{S_{E}}-\lambda_{I_{E}}\right)}{2 w_{3}}
$$

Hence, the required optimal control condition is obtained as

$$
u_{1}^{*}=\max \left(a_{1}, \min \left(b_{1}, \frac{S_{H}\left(\lambda_{S_{H}}-\lambda_{I_{H}}\right)}{2 w_{1}}\right)\right)
$$




$$
\begin{aligned}
& u_{2}^{*}=\max \left(a_{2}, \min \left(b_{2}, \frac{S_{M}\left(\lambda_{S_{M}}-\lambda_{I_{M}}\right)}{2 w_{2}}\right)\right) \\
& u_{3}^{*}=\max \left(a_{3}, \min \left(b_{3}, \frac{S_{E}\left(\lambda_{S_{E}}-\lambda_{I_{E}}\right)}{2 w_{3}}\right)\right)
\end{aligned}
$$

where $a_{1}, a_{2}, a_{3}=$ lower bounds and $b_{1}, b_{2}, b_{3}=$ upper bounds of the control variables $u_{1}, u_{2}$ and $u_{3}$ respectively.

\section{NUMERICAl Simulation}

Using the data given in Table 1, it is observed that $25 \%$ population gets infected by dengue disease, however approximately only $2 \%$ disease is transmitted from dengue free equilibrium point to the existence. In this section, we will analyze and study the effect of control on each compartment numerically.

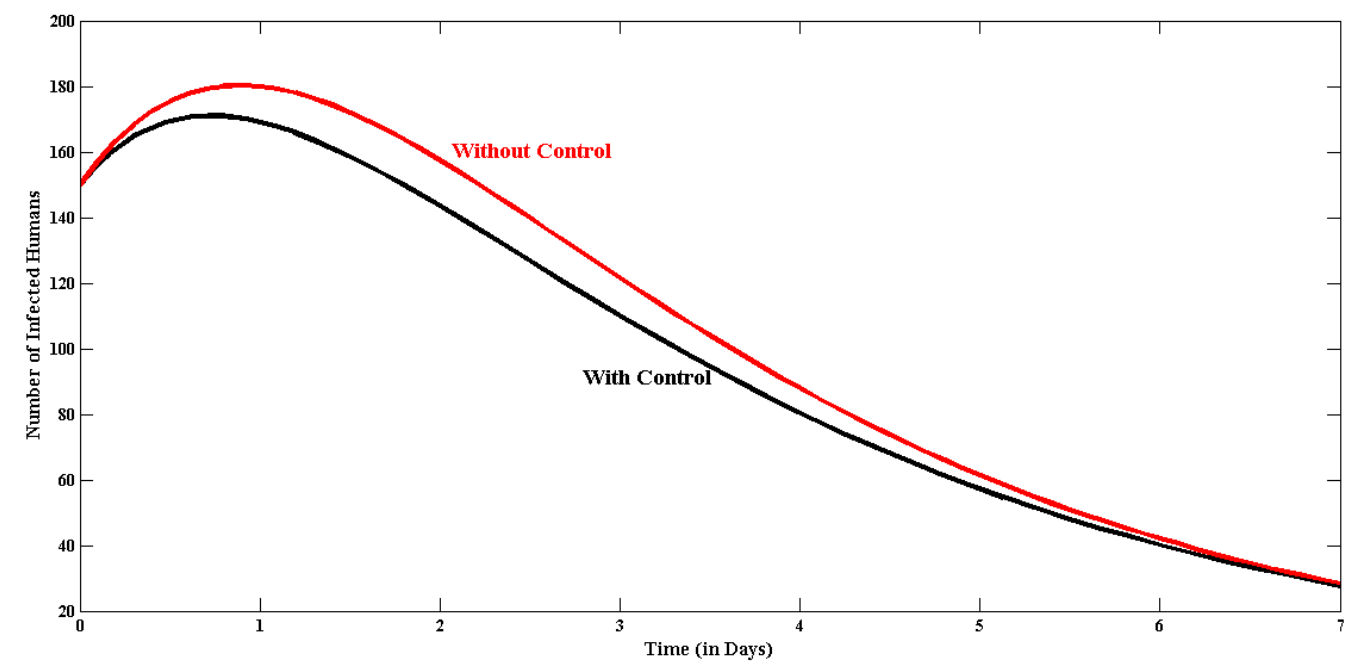

Figure 2. Control in terms of preventive measures on $I_{H}$ compartment

Fig. 2 depicts that when control is not applied infected humans increases from 150 to 180 but when control in terms of preventive measures are taken by them the individual decrease to 170 approximately within a day.

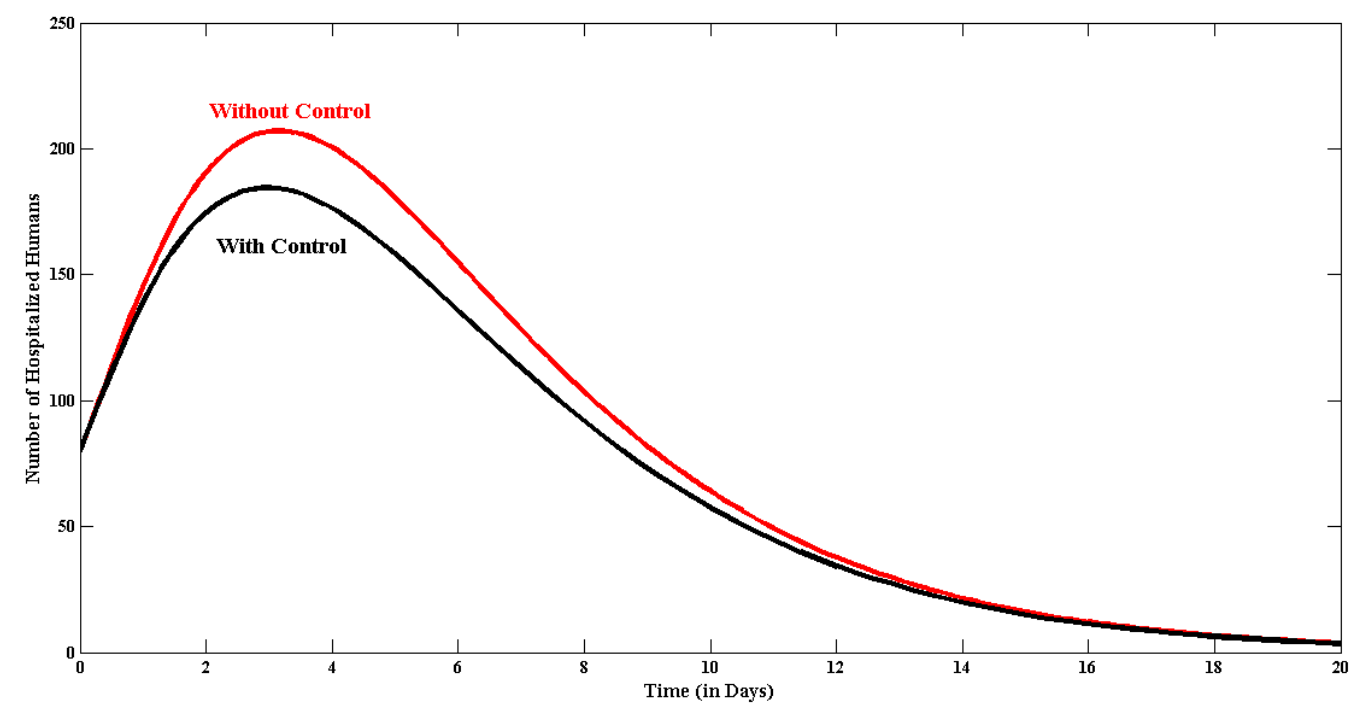

Figure 3. Control in terms of preventive measures on $H_{H}$ compartment

From Fig. 3 it can be seen that when an individual gets medical treatment along with preventive measures, they recover from the diseases faster comparatively. 


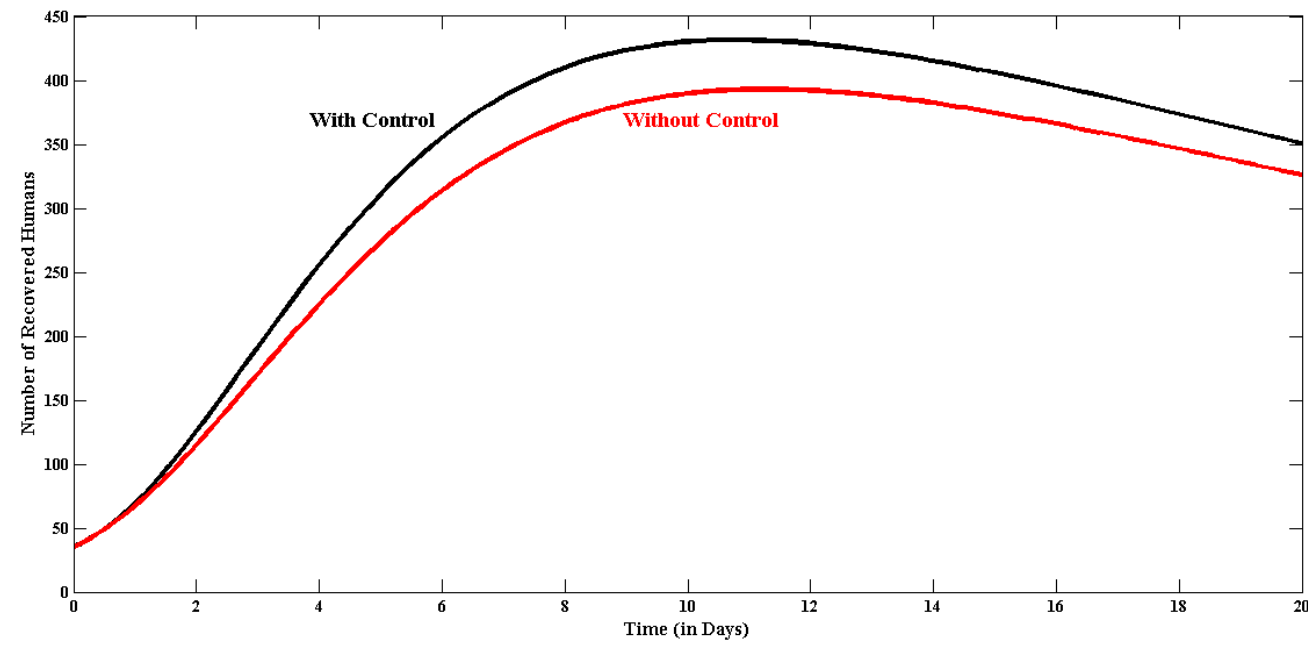

Figure 4. Control in terms of preventive measures on $R_{H}$ compartment

Fig. 4 shows that individual affected from dengue recovers soon if the ways to control are adopted by them. In fact, it seems to be more fruitful ass it increases the recovered individual from 390 to 430 in the same duration of 10 days.

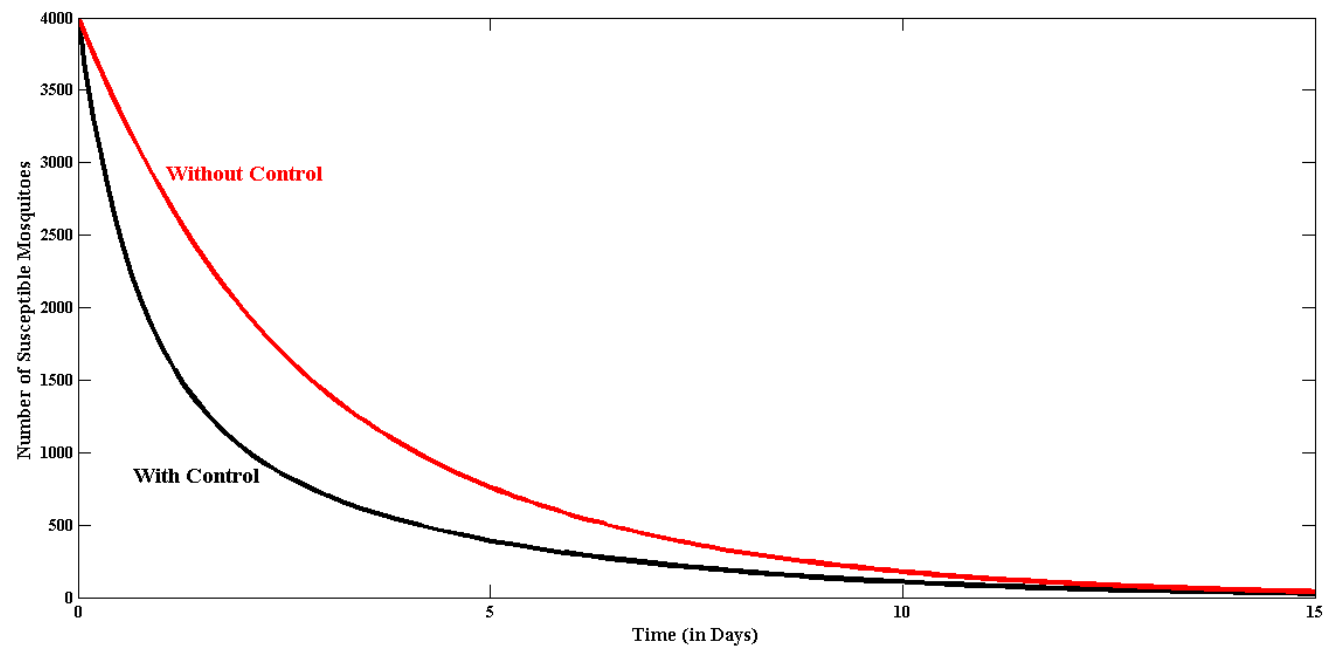

Figure 5. Control in terms of spraying insecticides on $S_{M}$ compartment

Fig. 5 shows that a mosquito responsible for dengue disease are removed from the community in a small duration of time when insecticides are used.

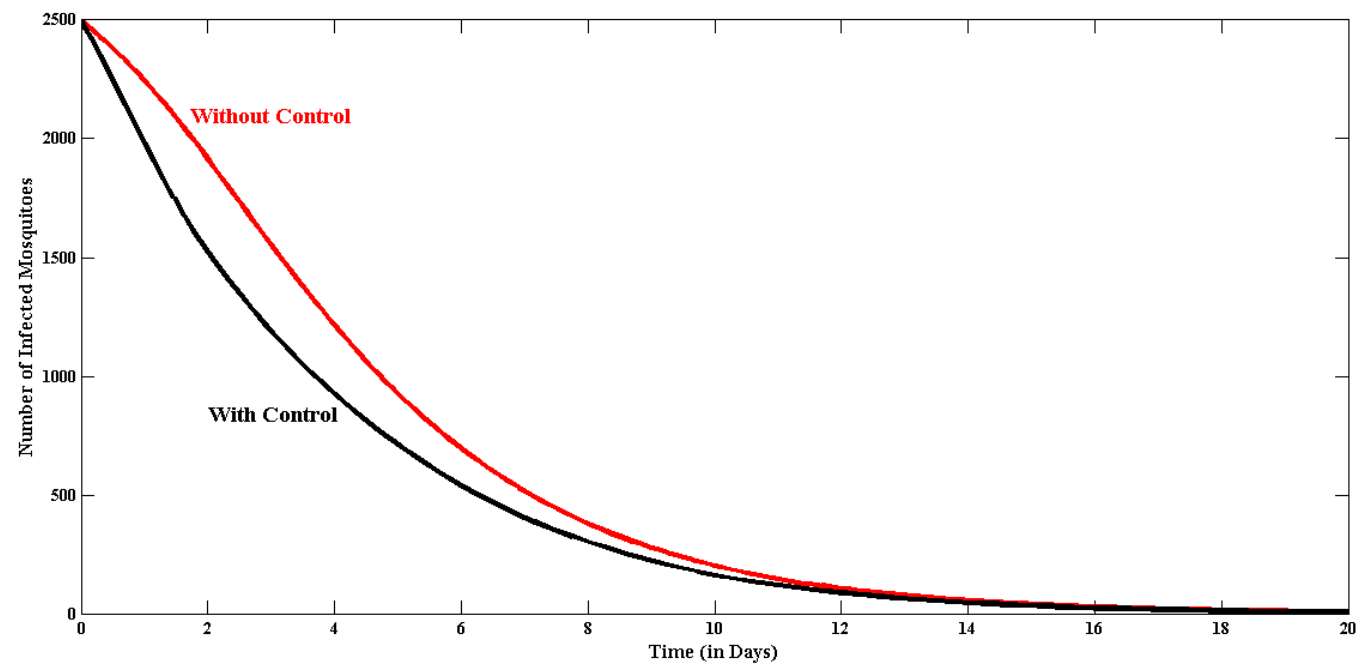

Figure 6. Control in terms of spraying insecticides on $I_{M}$ compartment 
Fig. 6 depicts that in the initial stage itself infected mosquitoes decreases in comparison to when control in terms of spraying insecticides is not done. But approximately after 12 days, no effect of control is observed which means that the mosquitoes have lost their life span and has died out.

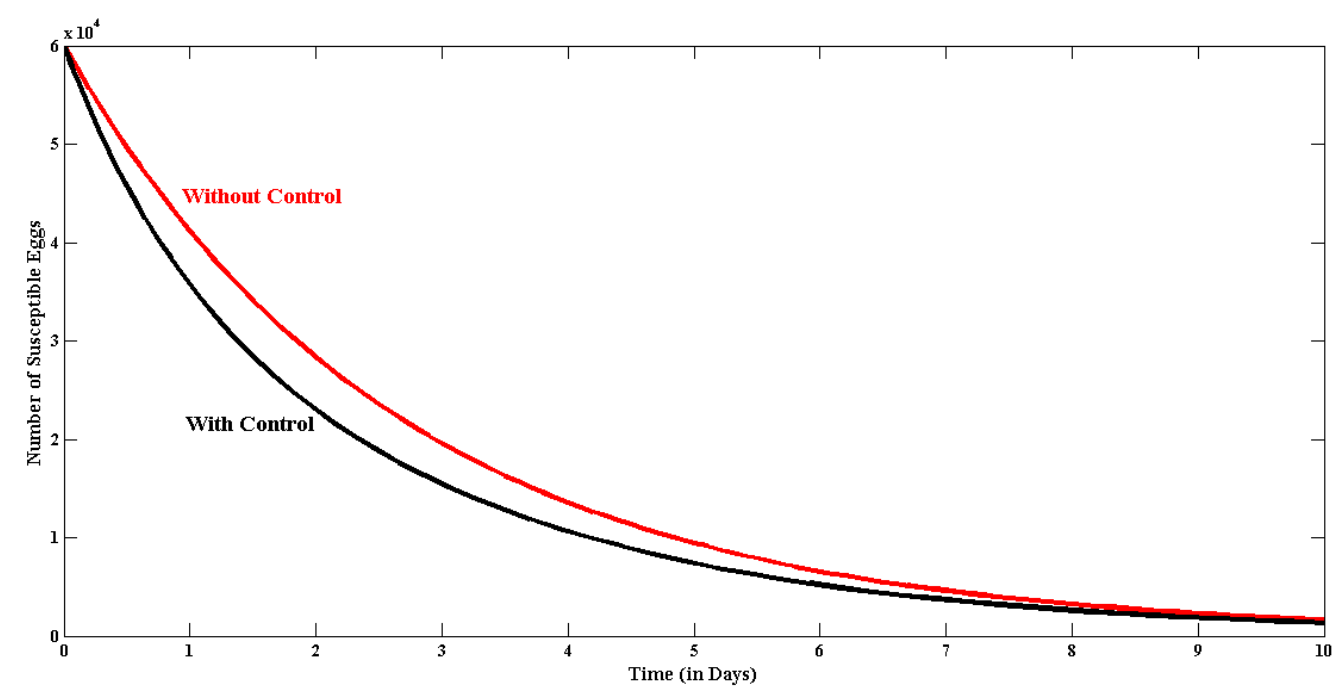

Figure 7. Control in terms of guppy fish on $S_{E}$ compartment

Fig. 7 shows that the eggs laid by a susceptible mosquito proves to be susceptible which can be decreased as this is the food of guppy fish. If this eggs are eaten by the fishes, we can have our surrounding free from mosquitoes.

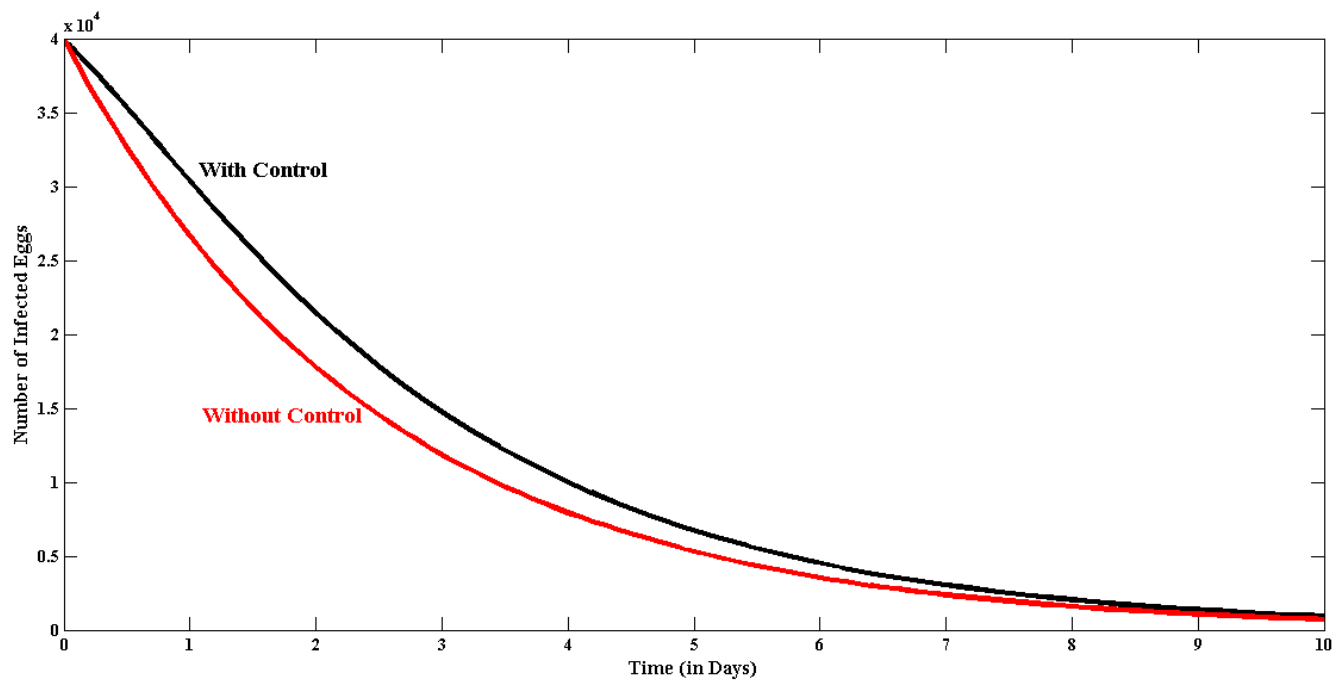

Figure 8. Control in terms of guppy fish on $I_{E}$ compartment

Fig. 8 depicts that the susceptible eggs which are not eaten by guppy fish will surely become infective and will give birth to an infected mosquito which is harmful for the individuals in the society. But it can be decreased by the control which is observed from the figure. 


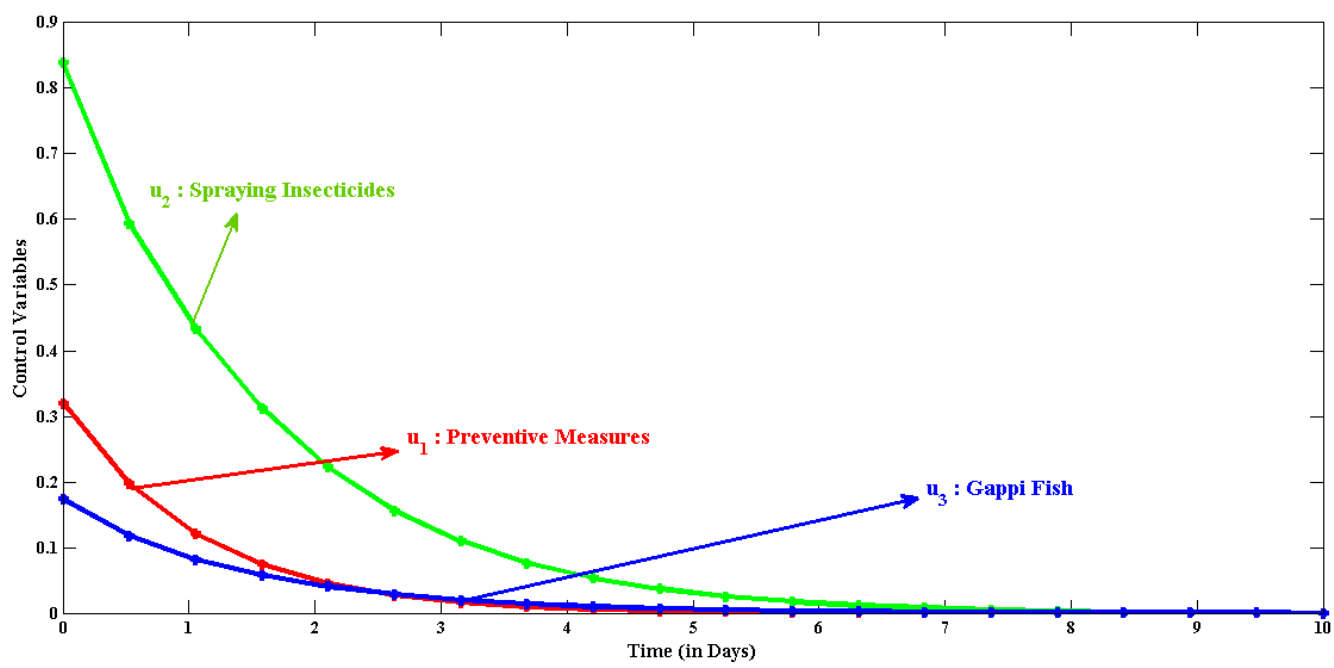

Figure 9. Control Variables versus Time (in days)

Fig. 9 shows that each control has a vital role in protecting humans from dengue disease. It is seen that $31 \%$ preventive measures, $83 \%$ use of insecticides, $18 \%$ Guppy fish are required in the initial days to stop oneself from becoming the victim of this disease.

\section{CONCLUSION}

In this paper, a mathematical model has been constructed to study the transmission of dengue disease. It has been established that this number of dengue affected individual can be reduced by using control on them as well on mosquitoes and their eggs. Two equilibrium points have been found: Dengue free equilibrium point and endemic equilibrium point. At both these points system proves to be locally asymptotically stable and globally asymptotically stable. Results for different compartments have been calculated numerically which interprets that to reduce overall severity of the dengue disease, increase in use of guppy fish at initial stage of mosquito cycle, increase spray of insecticides for the last stage of mosquito cycle and preventive measures at the end of humans is required.

\section{ACKNOWLEDGEMENTS}

The author thanks DST-FIST file \# MSI-097 for technical support to the Department of Mathematics.

\section{REFERENCES}

[1] https://en.wikipedia.org/wiki/Aedes Aegypti

[2] http://www.denguevirusnet.com/life-cycle-of-aedes-aegypti.html

[3] https://en.wikipedia.org/wiki/Dengue_fever

[4] https://www.webmd.com/a-to-z-guides/dengue-fever-reference\#1

[5] Asmaidi, A., Sianturi, P., and Nugrahani, E. H., A SIR Mathematical Model of Dengue Transmission and its Simulation, Indonesian Journal of Electrical Engineering and Computer Science. 12(11), 7920-7926, (2014).

[6] Diekmann, O., Heesterback, J.A.P., Roberts, M.G., The construction of next generation matrices for compartmental epidemic models, Journal of the Royal Society Interface, 7(47), 873-885, (2010).

[7] http://web.abo.fi/fak/mnf/mate/kurser/dynsyst/2009/R-Hcriteria.pdf

[8] LaSalle, J. P., The Stability of Dynamical Systems, Society for Industrial and Applied Mathematics, Philadelphia, Pa, (1976). https://doi.org/10.1137/1.9781611970432

[9] Fleming, W. H., Rishel, R. W., Deterministic and Stochastic optimal control (1). Springer Science and Business Media, (2012).

[10] Pontriagin, L.S., Boltyanskii, V.G., Gamkrelidze, R.V., Mishchenko, E. F., (1986). "The

Mathematical Theory of Optimal Process", Gordon and Breach Science Publishers, NY, USA, 4-5. 


\section{AUTHORS' BIOGRAPHY}
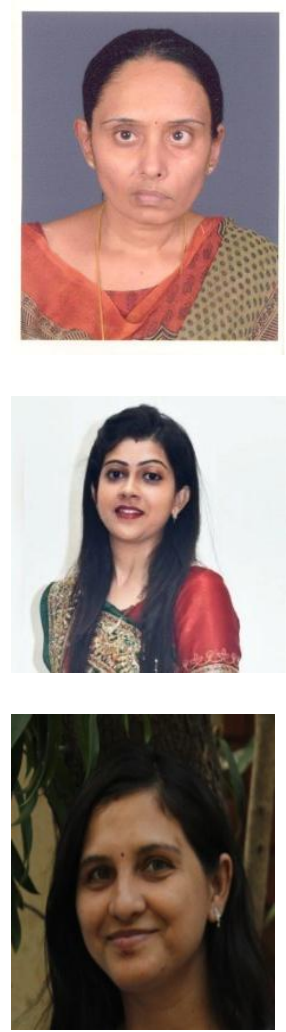

Nita H. Shah, is a Professor in the Department of Mathematics, Gujarat University, Ahmedabad. She has 25 years of research experience in inventory management, forecasting and information technology and information systems. She has published $375+$ articles in international journals, including APJOR (Singapore), International Journal of Production Economics, OMEGA, CCERO (Belgium), ECPE (Romania), Measuring, Control \& Simulation (France), JIOS (India), IJOMS (India), Industrial Engineering (India), European Journal of Operational Research, IJIR, IJOR, IJMOR, IJBPSCM, etc.

Foram Thakkar, is research scholar in department of mathematics, Gujarat University, India. She is working in dynamical systems of socio-medico problems. Her research interest is applications of mathematics in various disciplines. She has published 4- papers in JOBARI, AIR, DCDIS-B, ADSA.

Dr. Bijal Yeolekar, received Ph.D. recently in mathematics from Gujarat University, India. She worked in dynamical systems of social issues. Her research interest is applications of mathematics in various disciplines. She has published 18- papers in JOBARI, AIR, DCDIS-B, ADSA, IACM, AM, IJCM, $J E C E C S R$, etc.

Citation: N. H. Shah et al., " Simulation of Dengue Disease with Control ", International Journal of Scientific and Innovative Mathematical Research, vol. 5, no. 7, p. 14-28, 2017., http://dx.doi.org/10.20431/ 2347-3142.0507002

Copyright: (c) 2017 Authors. This is an open-access article distributed under the terms of the Creative Commons Attribution License, which permits unrestricted use, distribution, and reproduction in any medium, provided the original author and source are credited. 